\title{
Optical Communications and Networking Solutions for the Support of C-RAN in a 5G Environment
}

\author{
Vincenzo Eramo ${ }^{1, *}$, Marco Listanti ${ }^{1}$ and Francesco Giacinto Lavacca ${ }^{2}$ (D) \\ 1 DIET, University of Roma Sapienza, Via Eudossiana 18, 00184 Roma, Italy; marco.listanti@uniroma1.it \\ 2 Fondazione Ugo Bordoni, Viale del Policlinico 147, 00161 Roma, Italy; francescogiacinto.lavacca@uniroma1.it \\ * Correspondence: vincenzo.eramo@uniroma1.it; Tel.: +39-06-4458-5372
}

Received: 16 January 2019; Accepted: 24 January 2019; Published: 26 January 2019

check for updates

\section{Introduction}

The widespread availability of mobile devices such as tablets and smartphones has led to fast-increasing mobile data traffic in the last few years. Furthermore, based on different studies and predictions, it is possible to conclude that beyond 2020, mobile networks will be asked to support more than 1000 times today's traffic volume. Demands for higher mobile networks capacity, for increased data rates, and for a larger number of simultaneously connected devices are just a few of the requirements posed to the evolution of radio access networks. Other fundamental factors are energy saving and the cost of systems, latency, spectrum availability, and spectral efficiency. Naturally, one of the solutions to deal with the very high capacity and coverage demand is through strong radio site densification (e.g., through small, pico, femto cells), which could be also obtained by different deployment architectures. A Cloud Radio Access Network (C-RAN) or centralized RAN can be seen as a promising solution to deal with 5G requirements. Traditional C-RANs are organized as a three-element network: a distributed unit (DU), a central unit (CU), and a fronthaul network interconnecting the CU and DU [1]. The CU provides baseband signal processing functions and the DU provides the radio frequency (RF) signal transmission and reception functions. The C-RAN architecture enables Base Stations to be deployed flexibly and cost-effectively and is capable of performance enhancement through the use of coordinated multi-point transmission and reception (CoMP). The in-phase and quadrature (IQ) samples of the baseband signals are transmitted in the fronthaul network across a common public radio interface (CPRI). Ethernet-based technology [2] is probably the more promising and efficient option for the transport of CPRI traffic, but this technology is not yet mature and requires further investigation. For this reason, other solutions have been proposed such as the method based on Optical Transport Network (OTN) [3,4] and Dense Wavelength Division Multiplexing (DWDM) technologies, which are mature and allow for good performance in terms of the delay and jitter.

\section{Contributions}

The six papers published in this Special Issue propose and investigate new solutions and technologies to be applied in next-generation fronthaul networks.

Thirty-five authors from 16 institutions have contributed to the Special Issue. These institutions are located in Italy (4), Norway (1), Spain (3), Germany (1), China (1), Pakistan (1), Brazil (4), and Canada (1).

The first paper, authored by Eramo et al. and titled "Dimensioning Models of Optical WDM Rings in Xhaul Access Architectures for the Transport of Ethernet/CPRI Traffic" [5], proposes an Xhaul optical network architecture based on Optical Transport Network (OTN) and Dense Wavelength Division Multiplexing (DWDM) technologies. The network allows for a dynamic allocation of the 
bandwidth resources according to the current traffic demand. The network topology is composed of OTN/DWDM rings and the objective of the paper is to evaluate the best configuration (number of rings and number of wavelengths needed) to both to minimize the cost and to provide an implementable solution. The authors perform an analytical study and evaluate the advantages of the proposed dynamic resource allocation solution with respect to the static solution in which the network is provided with a number of wavelengths determined in the scenario in which the radio station works at full load. With this approach, the bandwidth saving can reach up to $90 \%$ in a $5 \mathrm{G}$ traffic scenario.

The second paper, authored by Mikaei et al. and titled "Traffic Estimation-Based Low-Latency XGS-PON Mobile Fronthaul for Small Cell C-RAN Based on Adaptive Learning Neural Network" [6], proposes and investigates a solution to guarantee low latency in fronthaul networks realized in Passive Optical Network (PON) technology. The proposed technique is based on the prediction of packets arriving at the Optical Network Unit (ONU) buffer from the Remote Radio Unit (RRU) using an adaptive learning neural network function integrated into the Dynamic Bandwidth Allocation (DBA) module at the optical line terminal (OLT). The performance of the new method is evaluated by means of simulation. The results confirm the capability of the proposed method of achieving the latency requirement for a mobile fronthaul network while outperforming algorithms proposed in the literature for the resource allocation in PON fronthaul networks.

The third paper, authored by Tonini et al. and titled "C-RAN Traffic Aggregation on Latency-Controlled Ethernet Links" [7], proposes and investigates a novel fronthaul network architecture that supports both CPRI traffic with a high time transparency and pre-emptiable backhaul traffic. The objective of the solution is to guarantee a hard delay for fronthaul traffic and at the same time to maximize the backhaul traffic throughput. The introduction of such a solution in a 5G transport network would allow compatibility with the widely deployed ethernet standard while significantly enhancing the scalability and the flexibility of the C-RAN optical infrastructure.

The fourth paper, authored by Ruiz et al. and titled "A Genetic Algorithm for VNF Provisioning in NFV-Enabled Cloud/MEC RAN Architectures" [8], proposes and investigates a C-RAN architecture provided with Network Function Virtualization (NFV) and Mobile Edge Computing (MEC) technologies in order to: (i) allow for a flexible processing and RAM resource allocation; (ii) guarantee better Quality of Service thanks to the availability of resources near to the user. The paper addresses the problems of Virtual Network Functions (VNF) provisioning (VNF-placement and service chain allocation) in a $5 \mathrm{G}$ network. In order to solve this problem, the authors propose a genetic algorithm that, considering both computing resources and optical network capacity, minimizes both the service blocking rate and processing resources usage. The proposed solution outperforms previous proposals in the literature, reducing the service blocking ratio while saving energy by reducing the number of active processing cores.

The fifth paper, authored by Mengesha and titled "Analysis of 5G New Radio Uplink Signals on an Analogue-RoF System Based on DSP-Assisted Channel Aggregation" [9], proposes and compares some solutions for the transmission of signals in 5G radio access technology on an interface referred to as New Radio (NR). The solutions are based on Digital Signal Processing (DSP)-assisted Analogue Radio-over-Fiber (A-RoF) transmission techniques. Frequency Division Multiple Access (FDMA) and Time Division Multiple Access (TDMA) channel aggregation techniques are considered and compared. The authors verify that $\sim 34 \%$ spectral efficiency gain and a lower Error Vector Magnitude (EVM) are achieved using the TDMA technique.

The sixth paper, authored by Frascolla et al. and titled "Optimizing C-RAN Backhaul Topologies: A Resilience-Oriented Approach Using Graph Invariants" [10], proposes and investigates resilient $5 \mathrm{G}$ access network topologies. The network topology design is based on graph theory. Specifically, the authors optimize some known graph invariants, such as the maximum node degree, topology diameter, average distance, and edge betweenness, as well as a new invariant called node Wiener impact, to achieve baseline network topologies that match the needs for $5 \mathrm{G}$ resilient future wireless and optical networks. 
Acknowledgments: This Special Issue has been made possible by the hard work of the authors. We want to than the reviewers and the MDPI assistant editors who, with their feedback, comments, and suggestions, have helped the authors to improve their papers. Finally, a special thanks goes to Ryan Pei for his continuous assistance and for motivating us day to day in organizing this Special Issue.

\section{References}

1. Eramo, V.; Listanti, M.; Lavacca, F.G.; Iovanna, P.; Bottari, G.; Ponzini, F. Trade-Off Between Power and Bandwidth Consumption in a Reconfigurable Xhaul Network Architecture. IEEE Access 2016, 4, 9053-9065. [CrossRef]

2. Chitimalla, D.; Kondepu, K.; Valcarenghi, L.; Tornatore, M.; Mukherjee, B. 5G Fronthaul Latency and Jitter Studies of CPRI Over Ethernet. J. Opt. Commun. Netw. 2017, 9, 172-182. [CrossRef]

3. Eramo, V.; Listanti, M.; Sabella, R.; Testa, F. Integrated OTN/WDM switching architecture equipped with the minimum number of OTN switches. J. Opt. Commun. Netw. 2014, 6, 138-151. [CrossRef]

4. Eramo, V.; Listanti, M.; Lavacca, F.G.; Sabella, R.; Testa, F. Performance evaluation of integrated OTN/WDM metropolitan networks in static and dynamic traffic scenarios. J. Opt. Commun. Netw. 2015, 7, 761-775. [CrossRef]

5. Eramo, V.; Listanti, M.; Lavacca, F.G.; Iovanna, P. Dimensioning Models of Optical WDM Rings in Xhaul Access Architectures for the Transport of Ethernet/CPRI Traffic. Appl. Sci. 2018, 8, 612. [CrossRef]

6. Mikaeil, A.M.; Hu, W.; Hussain, S.B.; Sultan, A. Traffic-Estimation-Based Low-Latency XGS-PON Mobile Front-Haul for Small-Cell C-RAN Based on an Adaptive Learning Neural Network. Appl. Sci. 2018, 8, 1097. [CrossRef]

7. Tonini, F.; Khorsandi, B.M.; Bjornstad, S.; Veisllari, R.; Raffaelli, C. C-RAN Traffic Aggregation on Latency-Controlled Ethernet Links. Appl. Sci. 2018, 8, 2279. [CrossRef]

8. Ruiz, L.; Duran, R.J.; de Miguel, I.; Khodashenas, P.S.; Pedreno-Manresa, J.J.; Merayo, N.; Aguado, J.C.; Pavon-Marino, P.; Siddiqui, S.; Mata, J.; et al. A Genetic Algorithm for VNF Provisioning in NFV-Enabled Cloud/MEC RAN Architectures. Appl. Sci. 2018, 8, 2614. [CrossRef]

9. Mengesha, B.D.; Torres-Ferrera, P.; Gaudino, R. Analysis of 5G New Radio Uplink Signals on an Analogue-RoF System Based on DSP-Assisted Channel Aggregation. Appl. Sci. 2019, 9, 47. [CrossRef]

10. Frascolla, V.; Dominicini, C.K.; Paiva, M.H.M.; Caporossi, G.; Marotta, M.A.; Ribeiro, M.R.N.; Segatto, S.E.V.; Martinello, M.; Monteiro, M.E.; Both, C.B. A Genetic Algorithm for VNF Provisioning in NFV-Enabled Cloud/MEC RAN Architectures. Appl. Sci. 2019, 9, 136. [CrossRef]

(C) 2019 by the authors. Licensee MDPI, Basel, Switzerland. This article is an open access article distributed under the terms and conditions of the Creative Commons Attribution (CC BY) license (http://creativecommons.org/licenses/by/4.0/). 